•生物编目・

\title{
被子植物APG分类系统评论
}

\author{
王 伟 1,2 张晓霞 ${ }^{1,2}$ 陈之端 ${ }^{1}$ 路安民 ${ }^{1 *}$ \\ 1 (中国科学院植物研究所系统与进化植物学国家重点实验室, 北京 100093) \\ 2 (中国科学院大学, 北京 100049)
}

\begin{abstract}
摘要: 随着植物分子系统学的兴起, 被子植物系统发育研究取得了举世瞩目的进展。被子植物系统发育组提出了 基于DNA证据的被子植物在目、科分类阶元上的分类系统, 简称APG系统。本文简要概括了APG系统的主要成就: (1)验证了被子植物分类系统的可重复性和可预言性; (2)解决了一些依据形态学性状未能确定的类群的系统位置; (3)证明了将被子植物一级分类分为双子叶植物和单子叶植物的不自然性; (4)证实了单沟花粉和三沟花粉在被子 植物高级分类单元划分中的重要性; (5)发现雄荵的向心发育和离心发育在多雄荵类群中是多次发生的, 不应作为 划分纲或亚纲的重要依据; (6)支持基于形态学(广义)性状划分的大多数科是自然的; (7)将一些长期认为自然的科 四分五裂。同时, 我们指出了尚需深入研究的几个问题: (1)如何将以分子数据建立的系统和以综合形态学证据建 立的系统相协调; (2)依据APG系统的研究结果需要创立新的形态演化理论; (3)只以“单系群”作为划分科、目的依据 值得商榷; (4)APG系统中一些目的分类没有可信的形态学共衍征; (5)依据APG系统需要做出一个自然系统的目、 科检索表和目、科的特征集要。此外, 我们对以亚洲, 特别是东亚为分布中心的一些类群的系统关系或分类等级 提出建议, 包括八角科、芒苞草科、水青树科、火筒树科、马尾树科、七叶树科、槭树科、伯乐树科应独立为科, 山茱英科(广义)应分为山茱英科(狭义)和蓝果树科(广义)。
\end{abstract}

关键词: 被子植物; 分子系统学; 分类; 形态学; 单系; 共衍征

\section{Comments on the APG's classification of angiosperms}

\author{
Wei Wang ${ }^{1,2}$, Xiaoxia Zhang ${ }^{1,2}$, Zhiduan Chen ${ }^{1}$, Anming Lu ${ }^{1 *}$ \\ 1 State Key Laboratory of Systematic and Evolutionary Botany, Institute of Botany, Chinese Academy of Sciences, Beijing \\ 100093 \\ 2 University of the Chinese Academy of Sciences, Beijing 100049
}

\begin{abstract}
With the rise of plant molecular systematics, tremendous progress has been made in understanding phylogenetic relationships within angiosperms. With the basic phylogenetic framework of angiosperms established, a DNA phylogeny-based angiosperm classification system at the order and familial levels was proposed by the Angiosperm Phylogeny Group (APG) in 1998 and has been updated three times. In this paper, we summarize the major achievements of the APG system as follows: (1) testing the repeatability and predictability of the APG system for angiosperms; (2) resolving the systematic positions of some segregate taxa which were not placed based on morphological characters; (3) proving that it is not reasonable to first divide angiosperms based on cotyledon character; (4) demonstrating the importance of tricolpate/tricolporate pollen and derivatives for angiosperm classification; (5) finding that the centrifugal development of stamens in polyandrous groups have evolved independently many times and should not be used to delimit class or subclass of angiosperms; (6) supporting that most of the families delimited by broad morphological characters are natural; and (7) separating some families which are traditionally regarded as natural. We then point out potential problems that need to be resolved in the future, including: (1) how to harmonize the APG system and the morphology-based systems; (2) establishing new morphological evolution theories on the basis of the APG system; (3) determining whether it is enough to only use "monophyly" as a criterion to circumscribe orders and families; (4) determining morphological synapormorphies of those orders in the APG sys-
\end{abstract}

收稿日期: 2017-01-13; 接受日期: 2017-03-15

基金项目: 国家自然科学基金(31470315, 31590822)、国家重点基础研究发展计划(2014CB954100)和中国科学院青年创新促进会专项

* 通讯作者 Author for correspondence. E-mail: anmin@ibcas.ac.cn 
tem; and (5) how to best compile a key to distinguish the orders and families of the APG system and to list their diagnostic characters for orders and families. In addition, we propose suggestions for the phylogenetic relationships and taxonomic status of some taxa mainly distributed in Asia, specifically East Asia, including Illiciaceae, Acanthochlamydaceae, Tetracentraceae, Leeaceae, Rhoipteiaceae, Hippocastenaceae, Aceraceae, Bretschneideraceae as familial status, and dividing Cornaceae sensu lato into Cornaceae sensu stricto and Nyssaceae sensu lato.

Key words: angiosperms; molecular systematics; classification; morphology; monophyly; synapormorphy

随着DNA测序和生物信息技术的发展，自 20 世纪90年代兴起利用分子数据研究生物类群间的 系统发育关系称为分子系统发育学(Molecular Phylogenetics)。在1993年, Mark Chase等42位作者合作 发表了“Phylogenetics of seed plants: an analysis of nucleotide sequences from the plastid gene $r b c L$ ”一文 (Chase et al, 1993)。这是由世界几十个实验室共同 完成的当时规模最大的系统发育分析, 在被子植物 系统学研究中具有划时代的意义。从那之后, 人们 逐渐广泛地利用DNA序列来重建植物类群间的系 统发育关系, 于是植物分子系统学作为植物系统学 的一个分支逐渐走向成熟。在1998年, 被子植物系 统发育组(Angiosperm Phylogeny Group, APG)综合 多个大尺度的系统发育分析结果, 为被子植物提出 了一个目、科分类阶元上的分类系统, 简称APG系 统。被子植物由此成为了第一个基于分子数据建立 分类系统的大类群。之后, 随着分子数据的增加, APG系统经历了3次修订(APG, 1998; APG II, 2003; APG III, 2009; APG IV, 2016)。该系统对被子植物系 统学和分类学研究产生了重大影响, 大大改变了两 百多年来植物学家们以形态学(广义)性状为根据提 出的分类系统。可以说, 最近20年在理解植物进化 历史上所取得的进展比过去200年都要大(Soltis et $\mathrm{al}, 2009)$ 。但并不是说APG系统已经完美, 不存在需 要继续研究的问题了。经历了两个多世纪的发展和 沉积, 人们积累了极为丰富的形态性状, 包括形态 学、解剖学、胚胎学、孢粉学、细胞学和个体发生 等, 这些性状在植物系统学和分类学研究中如何利 用和评价仍是当前亟待解决的课题。

本文就APG系统所取得的主要成就进行简要 归纳, 并提出一些尚需研究的问题。同时, 对以亚 洲, 特别是以东亚为分布中心的一些类群的系统关 系或分类等级提出建议。由于下面提到的每个方面 都是被子植物系统学的重要内容, 不可能用很短的
篇幅介绍其细节, 只是提纲彗领地谈谈我们的基本 观点和未来研究的方向。

\section{APG系统的主要成就}

\section{1 验证了被子植物分类系统的可重复性和可预 言性}

植物具有叶绿体、线粒体和核基因组三套遗传 体系, 不论来自哪个基因组的DNA信息都是可遗传 的。因此, 只要材料取样正确, 任何人在任何实验 室获得的DNA数据都应该是一样的, 从而所建立的 系统发育关系也是相同的。而对于形态学性状, 由 于不同作者在性状分析中对性状加权不同，或者相 同性状但排列顺序不同, 所建立的分类系统差异很 大。APG系统虽然经历了三次修订, 类群间的系统 发育关系解决得越来越好, 依据单系性的标准对目 的范围做了适当调整，但是强支持的目、科间的关 系一直是稳定的。一个自然的、能反映类群间系统 发育关系的分类系统, 才有可能根据一个分类群中 已知的性状(或类群)推测尚未发现的性状(或类群)。 如果一个相对稳定的分类系统有这样的预言价值, 那么人们为了研究植物的科或目之间的系统发育 关系而耗费时间和精力还是值得的( $\mathrm{Lu}, 1989)$ 。

\section{2 解决了一些依据形态学性状未能确定的类群 的系统位置}

被子植物在漫长的演化历史中演化出了一些 形态性状非常独特的类群, 导致不同的分类学家对 其提出的系统位置千差万别。例如, 领春木科 (Eupteleaceae)曾被归在木兰目(科) (Magnoliales/ Magnoliaceae) (Melchior, 1964)、金缕梅目 (Hamamelidales) (Thorne, 1992; Cronquist, 1981)或连香树 目 (Cercidiphyllales) (Dahlgren, 1983), Takhtajan (2009)甚至将其独立成目一一领春木目(Eupteleales)。 APG III将其放在毛茛目(Ranunculales)的基部(Wang et al, 2009)。独芯草科(Hydatelaceae)长期以来系统 
位置不确定, 被置于单子叶植物的禾本目(Poales) 附近。Saarela等(2007)利用多个DNA片段发现该科 是被子植物最基部的类群之一, 和睡莲目 (Nymphaeales)互为姐妹群。这一发现是继APG系统建立之 后植物分子系统学又一重大发现, 引起许多形态学 家重新研究独芯草科的形态, 并证实了该科和单子 叶植物没有直接的联系(Rudall et al, 2009)。APG III (2009)进一步扩大了睡莲目的系统范围, 将独荵草 科置于睡莲目中。

\section{3 证明了将被子植物一级分类分为双子叶植物 和单子叶植物的不自然性}

在传统分类系统中(如Cronquist, 1981; Dahlgren, 1983; Thorne, 1992; Takhtajan, 2009), 子叶数 目(1个或 2 个)被作为被子植物一级分类的性状。然 而, 分子系统学研究发现具双子叶的“ANITA进化 阶”(grade)构成被子植物最早分化的3个分支, 即无 油樟目(Amborellales)、睡莲目和木兰藤目(Austrobaileyales), 而单子叶植物与另外 4 个具双子叶的类 群一一金粟兰目(Chloranthales)、木兰类(Magnolids)、金鱼藻目(Ceratophyllales) 以及真双子叶植物 (Eudicots)构成被子植物早期快速辐射的五大分支 (图1; Moore et al, 2007)。

\section{4 证实了单沟花粉和三沟花粉在被子植物高级} 分类单元划分中的重要性

在基于形态的系统发育分析中, Donoghue和 Doyle (1989)发现以前被认为是双子叶植物的大量 物种组成了一个支持率很高的分支。这一分支由于 具有共衍征(synapomorphy)—三沟/三孔沟花粉或 其衍生物(tricolpate/tricolporate pollen and derivatives), 而被命名为 “三沟分支”(tricolpates; Donoghue \& Doyle, 1989)。后来的分子系统学研究都支持了该分 支的单系性(图1; Judd \& Olmstead, 2004)。Doyle和 Hotton (1991)将该分支命名为“真双子叶植物”, 并 获得了更广泛的应用。真双子叶植物是被子植物中 最大的分类群, 包括被子植物 300 多个科约 165,000 种(约占被子植物的64\%)。

1.5 发现雄芯的向心发育和离心发育在多雄芯类 群中是多次发生的, 不应作为划分纲或亚纲的重 要依据

多雄芯的向心发育和离心发育在传统分类上 曾被认为是一个非常重要的性状。Cronquist (1981)
和Takhtajan (2009)在他们的分类系统中将这一性状 作为划分纲或亚纲的依据之一。在APG系统中, 雄 芯的离心发育至少出现在10个目中(图1), 而且在这 些目中, 仅有非常少的类群具有离心发育的雄荵。 比如毛茛目, 仅毛茛科(Ranunculaceae)的耧斗菜属 (Aquilegia)的雄芯是向心发生、小孢子是离心发育 (冯旻等, 1995)。

\section{6 支持基于形态学(广义)性状划分的大多数科是 自然的}

在Cronquist (1981)系统中, 被子植物共包括 383科, 其中双子叶 318 科, 单子叶65科。在APG III (2009)中，约87\%的科的单系性得到支持，仅有 $13 \%$ 的科(约 50 个科)的界限和范围有大问题(Christenhusz et al, 2015), 如大戟科(Euphobiaceae)、大风子科 (Flacourtiaceae)、百合科(Liliaceae)、马齿苋科(Portulacaceae)和檀香科(Santalaceae)。而这些科多数在传 统分类上也被认为是异质的, 如广义百合科是一个 大杂烩, 后来曾被划分为30多个科(Dahlgren, 1983)。

\section{7 将一些长期作为自然的科四分五裂}

如玄参科的肢解: 玄参科(Scrophulariaceae)原 来拥有300属约5,000种(吴征镒等, 2003; Takhtajan, 2009)。依据分子数据, 许多属被归入车前科, 而半 寄生的属, 如马先蒿属(Pedicularis)被划到列当科 (Orobanchaceae)。APG系统的玄参科只包含52属 1,680种; 原来只有3属255种的车前科(Plantaginaceae) 现在拥有 104 属 1,820 种; 列当科由 15 属 210 种扩大 到96-99属2,060-2,100种(Stevens, 2001 onwards)。

\section{APG系统尚需研究的问题}

\section{1 如何将以分子数据建立的系统和以综合形态 学证据建立的系统相协调?}

APG IV (2016)将被子植物分为64目416科; Takhtajan (2009)分为156目560科。在APG系统的64 目中, 有19目与Takhtajan系统完全一致, 如樟目 (Laurales) (含7科)、鸭跖草目(Commelinales) (含5 科)、姜目(Zingiberales) (含8科)、桃金娘目(Myrtales) (含9科)和伞形目(Apiales) (含7科)等。有20余目由于 两大系统的目的概念不同, APG采取广义目而 Takhtajan采取狭义目, 但作为自然类群两个系统的 界定基本一致，如木兰目(Magnoliales)的6个科被 Takhtajan分在关系密切的 4 个目, 泽泻目(Alismatales) 13 个科在后者的 5 个目, 毛莨目 7 个科在后者的 7 个 


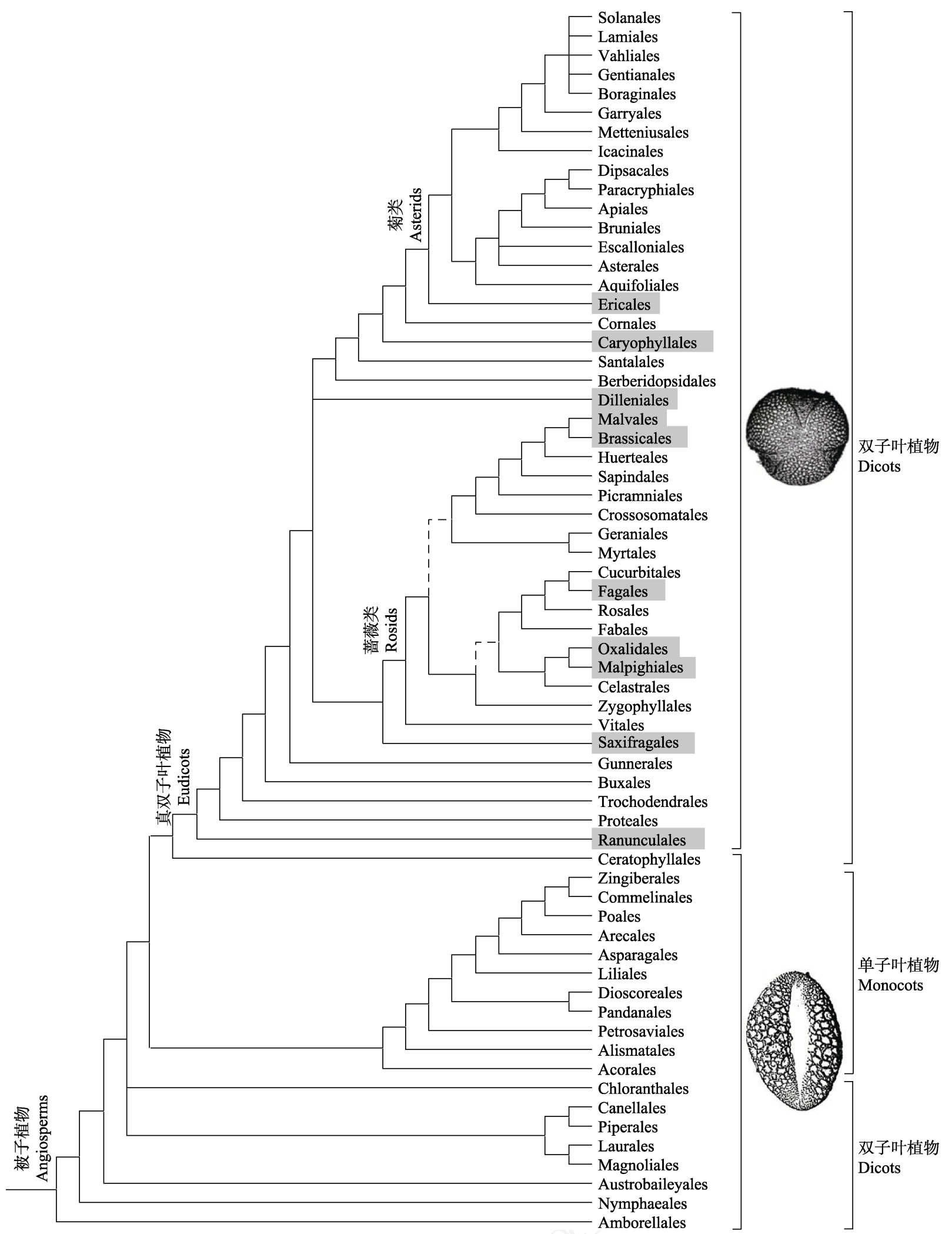

图1 被子植物APG IV (2016)系统的目间系统关系。虚线表示核/线粒体树与叶绿体树冲突; 标灰色的目含有多雄荵离心发育 的类群; 花粉和子叶性状标在系统树的右边。

Fig. 1 Interrelationships of the APG IV (2016) orders of angiosperms. The dotted lines indicate the conflicting placements between nuclear/mitochondrial and chloroplast trees. The orders with the gray contain at least one taxon with multiple centrifugal development stamens. Pollen and cotyledonal characters are labeled on the right. 
目, 杜鹃花目(Ericales)的 22 个科在后者的 12 个目等。

两大系统中科的归属分歧较大的目有 12 个, 如 薯蓣目(Dioscoreales) 3个科被Takhtajan (2009)归在 单子叶植物第 $1 、 9$ 和 21 目; 葫芦目 (Cucurbitales) 8 个科被归在双子叶植物第17、68、79、95、96目; 金 虎尾目(Malpighiales) 36 个科被归在双子叶植物第 17、35、50、52、53、66、77、82、86、94、99、 100 和 101 等 13 目 (图 2); APG 系统中有 4 目在 Takhtajan (2009)的系统中未设目而归于其他目, 如

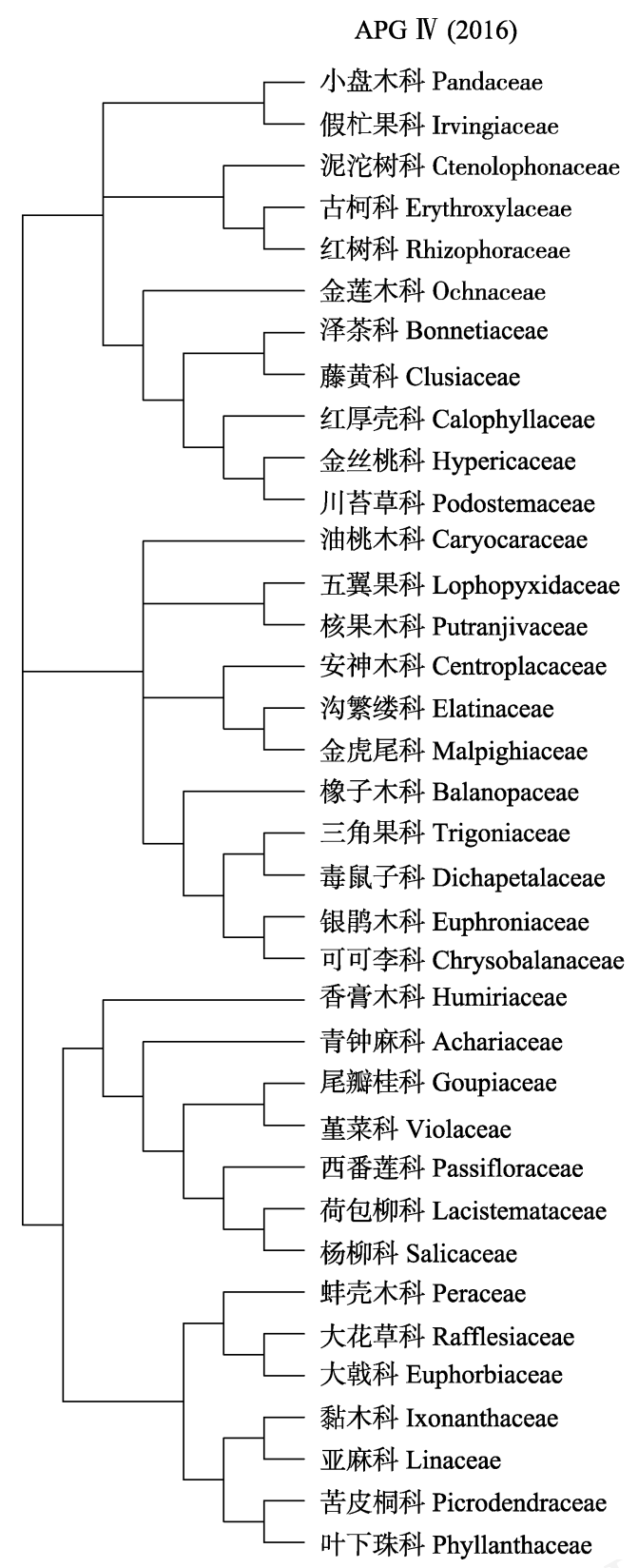

美洲苦木目(Picramniales)归于芸香目(Rutales)等。这 些目涉及了约 87 个科, 如何从形态学的角度理解这 些新的系统发育关系或分类等级尚需进一步研究。

\section{2 依据APG系统的研究结果需要创立新的形态} 演化理论

根据APG系统(图1), 被子植物最早分化的谱系 为无油樟目, 该目仅包括1种, 即无油樟(Amborella trichopoda Baill.), 它是雌雄异株的灌木, 其雌花有 2枚不育雄荵。第二谱系为水生的睡莲目, 而睡莲目

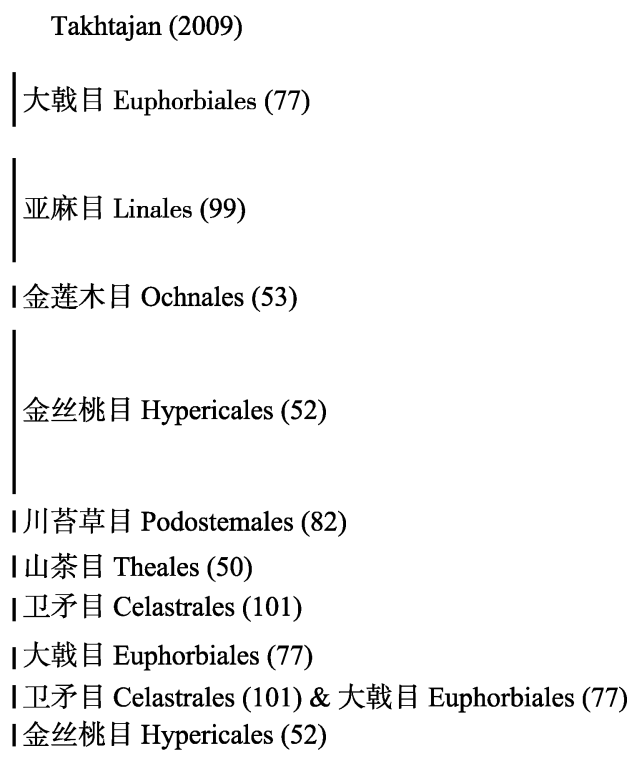

图2 比较APG IV (2016)系统与Takhtajan (2009)系统中金虎尾目的范围。括号中的数字示Takhtajan (2009)系统中目的序号。 Fig. 2 Comparison of the circumscription of Malpighiales between the APG IV (2016) and Takhtajan (2009) systems. The numbers in brackets indicate the serial numbers of the orders of Takhtajan (2009). 
最早分出的科是独芯草科。Rudall等(2009)将独荵草 科的生殖单元称为 “非花” (nonflower), 即有典型的 被子植物心皮和雄荵的结构, 但不能看作是典型的 花, 其“雄花” 只有单个雄荵, “雌花” 只有一个心皮, 生于同一“生殖单元”(reproductive units)的叫两性生 殖单元，生于不同的“生殖单元”叫单性生殖单元。 在两性生殖单元中, 雄芯(雄花)和心皮(雌花)的排 列是无序的。又如排列在ANITA之后的早期分化出 的金粟兰科(Chloranthaceae), 花极为简单: 单性花 的Ascarina的雄花只有1-3枚雄荵; 两性花的草珊瑚 属(Sarcandra)的“花”无花被, 只有苍片 1 枚、雄荵 1 枚、子房含 1 颗下垂的直生胚珠, 无花柱。该科可靠 的花和花粉化石发现于早白严世(Friis et al, 2011)。 此外, 在我国辽宁发现的早白严世的被子植物化石 古果科(Archaefructaceae)为水生草本, “花” 亦十分 简单(Sun et al, 2002)。由此, 我们认为水生植物在被 子植物演化早期就已分化, 并不都是从陆生植物演 化而来, 简单花不都是从复杂花简化来的, 单性花 也不都是从两性花退化而来的。即在被子植物起源 的早期, 水生、草本、简单花和单性花就已经出现了, 这就对传统的“真花说”和“假花说” 都提出了挑战。

\section{3 只以“单系群”作为划分目、科的依据值得商榷}

APG系统划分目、科最重要的标准就是单系性 (monophyly; Chase et al, 2000)。但由于取样的不完 全和物种绝灭等造成的间断, “并系群”(paraphyletic group) 是客观存在的。按照进化系统学家的观点, “并系群”也是自然类群(Stuessy, 2010)。单系性的标 准使得传统上一些目、科的范围发生了变化, 这给 实际运用带来了困难。比如, APG系统将㵞科(Chenopodiaceae) 归并到苋科(Amaranthaceae)之中, 但 是荺科本身还是一个单系群, 只是镶嵌在苋科之 中。而传统分类上的藜科和苋科是容易区别的, 在 其他生物学领域也是广泛接受的。

\subsection{APG系统的一些目或科没有可信的形态学共 衍征}

由于严格强调单系性, APG系统中一些目、科 比较异质, 其共衍征很难确定。例如, 由莲科 (Nelumbonaceae)、悬铃木科(Platanaceae)、山龙眼 科(Proteaceae)组成的山龙眼目(Proteales) (APG III, 2009), 很难找到它们的共衍征, 而APG IV (2016) 又把清风藤科(Sabiaceae)归入到山龙眼目, 这导致 该目更为异质。又如, APG系统的金虎尾目是一个
大拼盘，包含的36个科分布在Takhtajan (2009)系统 中不同位置的13个目中(图2)。分化时间分析显示金 虎尾目的28个主要分支在白严纪中期(112-94 Ma) 由爆发式的快速辐射所产生(Davis et al, 2005)。此 外, APG III (2009)界定的虎耳草目(Saxifragales)包 括了 Takhtajan (2009)的29、31、33、34、66和81目(图 3)。通过仔细研究形态性状的进化, Carlsward等 (2011)发现虎耳草目的共衍征为叶具堇菜型(violoid) 或山茶型(theoid)叶齿(图3)。APG IV (2016)依据S. Bellot和S. Renner未发表的数据将寄生植物科锁阳科(Cynomoriaceae)放在虎耳草目中, 而锁阳科 在 Takhtajan 系统中独立成目一锁阳目 (Cynomoriales) (第103目)。近来Bellot等(2016)根据 叶绿体、线粒体和核基因组的数据将锁阳科放在虎 耳草目的最基部, 这导致Carlsward等(2011)所发现 的叶的性状不能用于界定扩大的虎耳草目(图3)。上 述分析说明APG系统中一些目, 如睡莲目、山龙眼 目、金虎尾目、虎耳草目以及蓄薇目(Rosales)等的划 分仍需研究。

\section{5 依据APG系统需要做出一个自然系统的目、科} 检索表和目、科的特征集要

APG系统是根据分子数据提出的分类系统。当 前, 它仍然缺少一个目、科水平的自然检索表, 这 限制了它的广泛使用。同时, 目、科的鉴别特征鲜 有描述。因此, 未来需要综合形态和分子证据, 尤 其是利用分子系统发育框架, 在大尺度上分析各种 形态性状的进化式样, 确定各目、科的鉴别性状 (diagnostic character), 从而提出APG系统的目、科 检索表, 及其目、科的特征集要。

\section{3 对亚洲, 特别是东亚为分布中心的一些 类群系统关系或分类等级的建议}

Christenhusz等(2015)公布了他们就“被子植物 和硕类植物科的界限”对世界上 42 个国家或地区的 441位参与者在线的调查结果。参加者中, 北美155 人(其中美国 139 人), 中南美洲 24 人(其中巴西6人), 欧洲 141 人(其中英国66人、法国20人、德国 13 人)、 非洲 13 人(其中南非 10 人)、亚洲 27 人(其中中国大陆 6 人、台湾地区6人)、澳大利亚-太平洋地区43人(其 中澳大利亚 35 人、新西兰 7 人)。显然, 这次调查选 择的植物类群和参与者带有明显的地域偏见, 对在 世界上植物种类最丰富的中国, 大陆只有 6 人, 连 


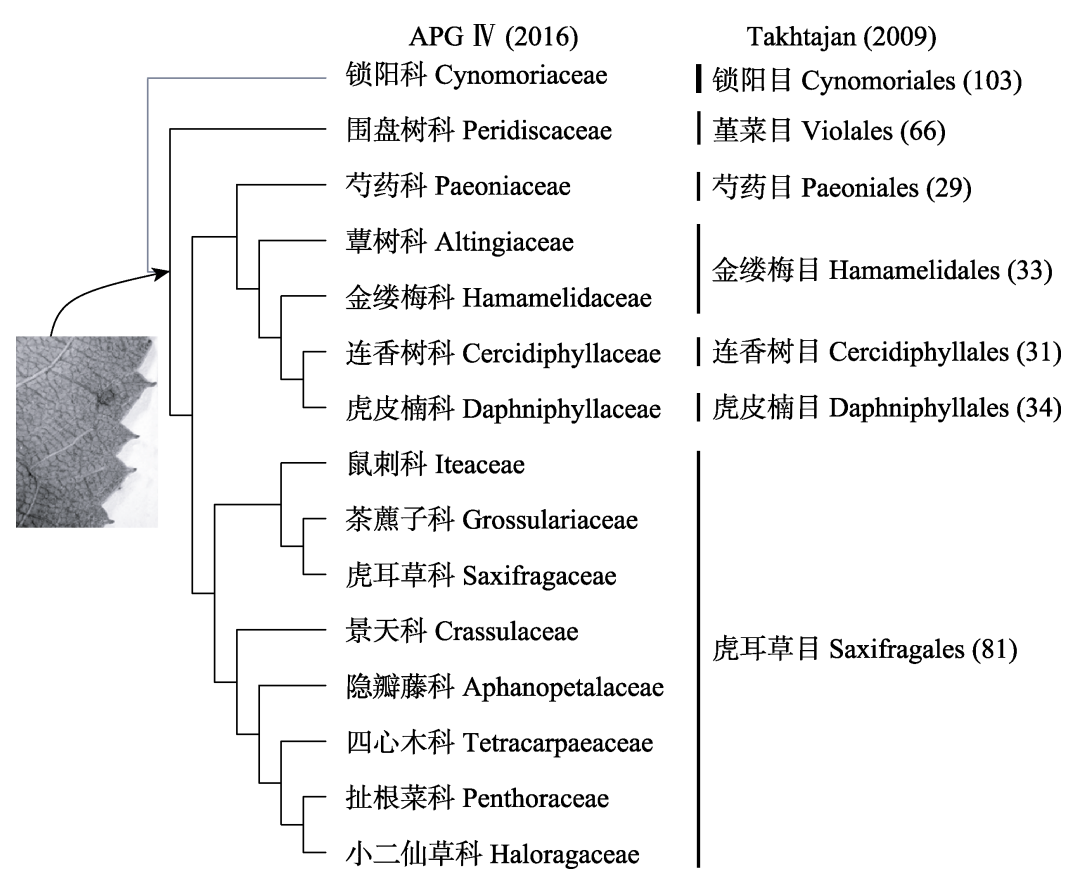

图3 比较APG IV(2016)系统与Takhtajan(2009)系统中虎耳草目的范围。锁阳科的位置根据Bellot等(2016)的结果。括号中的 数字示Takhtajan(2009)系统中目的序号。

Fig. 3 Comparison of the circumscription of Saxifragales between the APG IV (2016) and Takhtajan (2009) systems. The placement of Cynomoriaceae is based on the result of Bellot et al (2016). The numbers in brackets indicate the serial numbers of the orders of Takhtajan (2009).

同台湾也仅 12 人参加, 而中国植物分类学家独立自 主编著出版了世界上规模最大、包含种类最多、含 80 卷126册的《中国植物志》, 并作为各科的第一作 者合作出版了 25 卷Flora of China。实际上, APG系 统对于以亚洲为分布中心的一些科的划分十分不 妥。举例如下:

(1)八角科(Illiciaceae)和五味子科(Schisandraceae)。APG系统将八角科归并到五味子科。分子系 统学研究显示传统的八角科和五味子科均为单系 群, 呈姐妹群关系(Stevens, 2010 onwards)。而两科 的习性、花果的形态学、木材解剖和化学成分等性 状显著不同(吴征捡等, 2003; Takhtajan, 2009)。因此, 将它们作为独立科组成八角目(Illiciales)比较恰当。

(2)芒苍草科(Acanthochlamydaceae)和翡若翠科 (Velloziaceae)。APG系统将芒苞草科归并到翡若翠 科。翡若翠科含 8 属 250 种, 间断分布于中南美洲的 巴拿马到阿根廷, 尤其在巴西东南部和非洲及阿拉 伯西南部。单型的芒苞草科局限分布于中国横断山 中段海拔2,700-3,500 m的亚高山河谷。根据综合的 形态学性状(吴征镒等, 2003; Takhtajan, 2009)和分 子证据及隔离的生境和分布, 应支持芒苞草科为独
立科。

(3)水青树科(Tetracentraceae)和昆栏树科(Trochodendraceae)。APG系统将两科合并为昆栏树科, 由两个十分古老的单型属昆栏树属(Trochodendron) 和水青树属(Tetracentron)组成, 两者均为东亚-喜 马拉雅特有, 它们在生长习性、营养器官、花结构、 胚胎学、染色体等性状(吴征镒等, 2003; Takhtajan, 2009)已相当分化，足已将它们分别独立成科。

(4)火筒树科 (Leeaceae)和葡萄科 (Vitaceae)。 APG系统将火筒树科归并到葡萄科。火筒树属(Leea) 有34种, 旧世界热带分布, 有一组十分特别的形态 性状, 如习性为直立乔、灌至草本, 无卷须, 花瓣基 部下联合成柱等不同于葡萄科的其他属(吴征捡等, 2003; Takhtajan, 2009), 以单独成科为佳。

(5) 马尾树科 (Rhoipteiaceae)和胡桃科 (Juglandaceae)。APG系统将马尾树科归并到胡桃科。马尾 树科是Handel-Mazzetii (1932)发表的单型科, 产于 我国贵州南部及东南部、云南东南部、广西北部至 西部和越南北部, 形态独特, 曾被置于菖麻目(Urticales)。经多学科综合研究, 认为它接近胡桃科, 但 比胡桃科原始(路安民和张志耘，1990; Zhang et al, 
1994; 吴征镒等, 2003; Takhtajan, 2009)。分化时间 分析显示马尾树科与胡桃科在晚白严世( 80 Ma)就 已经分开(Xiang et al, 2014)。因此, 马尾树科仍应独 立成科。

(6)七叶树科(Hippocastenaceae)、棫树科(Aceraceae)和无患子科(Sapindaceae)。APG系统将七叶 树科和槭树科归并到无患子科, 认为如果无患子科 不包括前2科, 其他属就形成一个并系群。实际上, 在广义无患子科的分支分析中(Judd et al, 1994; Harrington et al, 2005)得到4个较好支持的分支: 第 一支是七叶树科分支; 第二支是传统的槭树科分支 (包括槭树属(Acer)和金钱槭属(Dipteronia)); 第三 支是车桑子支; 第四支是传统的无患子科。这 4 个分 支在形态学上有明显的区别, 因此应当将传统上的 七叶树科和槭树科独立成科, 在分布区类型上, 该 2 科属温带分布, 无患子科(狭义)为热带分布(吴征 镒等, 2003; Takhtajan, 2009)。

(7)伯乐树科 (Bretschneideraceae)和叠桑树科 (Akaniaceae)。APG系统将中国准特有的伯乐树科归 并到澳大利亚东部特有的单型科——叠桑树科。在 Takhtajan (2009)所列出的大段的检索特征足以将它 们分成不同的科; 加之两科南北半球间断分布。近 来分化时间计算显示两者在早古新世( 64 Ma)就已 分开(Cardinal-McTeague et al, 2016), 表明它们有很 古老的祖先, 呈子遗状态。

(8)桃叶珊瑚科 (Aucubaceae) 和丝缨花科 (Garryaceae)。APG系统将东亚特有的单属科桃叶珊瑚 科归并到美国西部特有的单属科丝缆花科。Takhtajan (2009)详细地列出了这两科在花部形态、胚胎 学、细胞学等方面显著不同的性状, 应当分立, 作 为姐妹科放在丝缨花目(Garryales)。

(9)山荣莫科(Cornaceae)的范围。APG III系统的 山茱英科包括了八角枫科(Alangiaceae)、蓝果树科 (Nyssaceae)、单室荣英科(Mastixiaceae)、珙桐科 (Davidiaceae)以及狭义的山荣英科。吴征镒等(2003) 和Takhtajan (2009)将它们各自立科。Chen等(2016) 根据5个基因( $a t p B 、 m a t K 、 n d h F 、 r b c L$ 和 $m a t R)$ 的分 析, 发现山荣英属(Cornus) + 八角枫属(Alangium) 为一支; 蓝果树属 $($ Nyssa $)+$ 喜树属(Camptotheca) + 珙桐属(Davidia) + 马蹄参属(Diplopanax $)+$ 单 室荣英属(Mastixia)为一支, 两个大支都有 $100 \%$ 的
支持率。因此，应将广义的山荣英科分为山荣英科 (狭义, 包括八角枫属)和蓝果树科(广义)。

\section{参考文献}

APG (1998) An ordinal classification for the families of flowering plants. Annals of the Missouri Botanical Garden, 85, 531-553.

APG II (2003) An update of the Angiosperm Phylogeny Group classification for the orders and families of flowering plants: APG II. Botanical Journal of the Linnean Society, 141, 399-436.

APG III (2009) An update of the Angiosperm Phylogeny Group classification for the orders and families of flowering plants: APG III. Botanical Journal of the Linnean Society, 161, 105-121.

APG IV (2016) An update of the Angiosperm Phylogeny Group classification for the orders and families of flowering plants: APG IV. Botanical Journal of the Linnean Society, 181, 1-20.

Bellot S, Cusimano N, Luo S, Sun G, Zarre S, Gröger A, Temsch E, Renner S (2016) Assembled plastid and mitochondrial genomes, as well as nuclear genes, place the parasite family Cynomoriaceae in the Saxifragales. Genome Biology and Evolution, 8, 2214-2230.

Cardinal-McTeague WM, Sytsma KJ, Hall JC (2016) Biogeography and diversification of Brassicales: a 103 million year tale. Molecular Phylogenetics and Evolution, 99, 204224.

Carlsward BS, Judd WS, Soltis DE, Manchester S, Soltis PS (2011) Putative morphological synapomorphies of Saxifragales and their major subclades. Journal of the Botanical Research Institute of Texas, 5, 179-196.

Chase MW, Fay MF, Savolainen V (2000) Higher-level classification in the angiosperms: new insights from the perspective of DNA sequence data. Taxon, 49, 685-704.

Chase MW, Soltis DE, Olmstead RG, Morgan D, Les DH, Mishler BD, Duvall MR, Price R, Hills HG, Qiu Y-L, Kron KA, Retting JH, Conti E, Palmer JD, Manhart JR, Sytsma KJ, Michaels HJ, Kress WJ, Karol KG, Clark WD, Hedren M, Gaut BS, Jansen RK, Kim K-J, Wimpee CF, Smith JF, Furnier GR, Strauss SH, Xiang Q-Y, Plunkett GM, Soltis PS, Williams SE, Gadek PA, Quinn CJ, Eguiarte LE, Golenberg E, Learn GH, Graham S, Barrett SCH, Dayanandan S, Albert VA (1993) Phylogenetics of seed plants: an analysis of nucleotide sequences from the plastid gene $r b c L$. Annals of the Missouri Botanical Garden, 80, 528-580.

Chen ZD, Yang T, Lin L, Lu LM, Li HL, Sun M, Liu B, Chen M, Niu YT, Ye JF, Cao ZY, Liu HM, Wang XM, Wang W, Zhang JB, Meng Z, Cao W, Li JH, Wu SD, Zhao HL, Liu ZJ, Du ZY, Wang QF, Guo J, Tan XX, Su JX, Zhang LJ, Yang LL, Liao YY, Li MH, Zhang GQ, Chung SW, Zhang J, Xiang KL, Li RQ, Soltis DE, Soltis PS, Zhou SL, Ran JH, 
Wang XQ, Jin XH, Chen YS, Gao TG, Li JH, Zhang SZ, Lu AM, China Phylogeny Consortium (2016) Tree of life for the genera of Chinese vascular plants. Journal of Systematics and Evolution, 54, 277-306.

Christenhusz MJM, Vorontsova MS, Fay MF, Chase MW (2015) Results from an online survey of family delimitation in angiosperms and ferns: recommendations to the Angiosperm Phylogeny Group for thorny problems in plant classification. Botanical Journal of the Linnean Society, 178, 501-528.

Cronquist A (1981) An Integrated System of Classification of the Flowering Plants. Columbia University Press, New York.

Dahlgren R (1983) General aspects of angiosperm evolution and macro-systematics. Nordic Journal of Botany, 3, 119-149.

Davis CC, Webb CO, Wurdack KJ, Jaramillo CA, Donoghue MJ (2005) Explosive radiation of Malpighiales supports a Mid-Cretaceous origin of modern tropical rain forests. The American Naturalist, 165, E36-E65.

Donoghue MJ, Doyle JA (1989) Phylogenetic analysis of angiosperms and the relationships of Hamamelidae. In: Evolution, Systematics, and Fossil History of the Hamamelidae, vol. 1 (eds Crane PR, Blackmore S), pp. 17-45. Clarendon Press, Oxford, UK.

Doyle JA, Hotton CL (1991) Diversification of early angiosperm pollen in a cladistic context. In: Pollen and Spores: Patterns of Diversification (eds Blackmore S, Barnes SH), pp. 165-195. Clarendon Press, Oxford, UK.

Feng M, Fu DZ, Liang HX, Lu AM (1995) Floral morphogenesis of Aquilegia L. (Ranunculaceae). Acta Botanica Sinica, 37, 791-794. (in Chinese with English abstract) [冯 旻, 傅德志, 梁汉兴, 路安民 (1995) 耧斗菜属花部形态 发生. 植物学报, 37, 791-794.]

Friis EM, Crane PR, Pedersen KR (2011) Early Flowers and Angiosperm Evolution. Cambridge University Press, Cambridge, UK.

Handel-Mazzetti H (1932) Rhoipteaceae, eine nenu Familie der Monochlamydeen. Feddes Repertorium, 30, 75-80.

Harrington MG, Edwards KJ, Johnson SA, Chase MW, Gadek PA (2005) Phylogenetic inference in Sapindaceae sensu lato using plastid matK and $r b c \mathrm{~L}$ DNA sequences. Systematic Botany, 30, 366-382.

Judd WS, Olmstead RG (2004) A survey of tricolpate (eudicot) phylogenetic relationships. American Journal of Botany, 91, 1627-1644.

Judd WS, Sanders RW, Donoghue MJ (1994) Angiosperm family pairs: preliminary phylogenetic analyses. Harvard Papers in Botany, 5, 1-51.

Lu AM (1989) Explanatory notes on R. Dahlgren's system of classification of the angiosperms. Cathaya, 1, 149-160.

Lu AM, Zhang ZY (1990) The differentiation, evolution and systematic relationship of Juglandales. Acta Phytotaxonomica Sinica, 28, 96-102. (in Chinese with English ab- stract) [路安民, 张志耘 (1990) 胡桃目的分化、进化和系 统关系. 植物分类学报, 28, 96-102.]

Melchior H (1964) A. Engler's Syllabus der Pflanzenfamilien Band II. Gebrüder Borntraeger, Berlin-Nikolassee.

Moore MJ, Bell CD, Soltis PS, Soltis DE (2007) Using plastid genome-scale data to resolve enigmatic relationships among basal angiosperms. Proceedings of the National Academy of Sciences, USA, 104, 19363-19368.

Rudall PJ, Remizowa MV, Prenner G, Prychid CJ, Tuckett RE, Sokoloff DD (2009) Nonflowers near the base of extant angiosperms? Spatiotemporal arrangement of organs in reproductive units of Hydatellaceae and its bearing on the origin of the flower. American Journal of Botany, 96, 67-82.

Saarela JM, Rai HS, Doyle JA, Endress PK, Mathews S, Marchant AD, Briggs BG, Graham SW (2007) Hydatellaceae identified as a new branch near the base of the angiosperm. Nature, 446, 312-315.

Soltis DE, Moore MJ, Burleigh G, Soltis PS (2009) Molecular markers and concepts of plant evolutionary relationships: Progress, promise and future prospects. Critical Reviews in Plant Sciences, 28, 1-15.

Stevens PF (2001 onwards) Angiosperm Phylogeny Website. Version 12, July 2012. http: //www.mobot.org/MOBOT/research/APweb/ (accessed on 2017-01-07).

Stuessy TF (2010) Paraphyly and the origin and classification of angiosperms. Taxon, 59, 689-693.

Sun G, Ji Q, Dilcher DL, Zheng S, Nixon KC, Wang X (2002) Archaefructaceae, a new basal angiosperm family. Science, 296, 899-904.

Takhtajan A (2009) Flowering Plants, 2nd edn. Springer, Heidelberg.

Thorne RF (1992) Classification and geography of the flowering plants. Botantical Review, 58, 225-348.

Wang W, Lu AM, Ren Y, Chen ZD (2009) Phylogeny and classification of Ranunculales: evidence from four molecular loci and morphological data. Perspectives in Plant Ecology, Evolution and Systematics, 11, 81-110.

Wu ZY, Lu AM, Tang YC, Chen ZD, Li DZ (2003) The families and genera of angiosperms in China: a comprehensive analysis. Science Press, Beijing. (in Chinese) [吴征镒, 路安 民, 汤彦承, 陈之端, 李德铢 (2003) 中国被子植物科属 综论. 科学出版社, 北京.]

Xiang XG, Wang W, Li RQ, Lin L, Liu Y, Zhou ZK, Li ZY, Chen ZD (2014) Large-scale phylogenetic analyses reveal fagalean diversification promoted by the interplay of diaspores and environments in the Paleogene. Perspectives in Plant Ecology, Evolution and Systematics, 16, 101-110.

Zhang ZY, Lu AM, Wen J (1994) Embryology of Ehoiptelea chiliantha (Rhoipteaceae) and its systematic relationship. Cathaya, 6, 57-66.

(责任编委：高连明 责任编辑：时意专) 